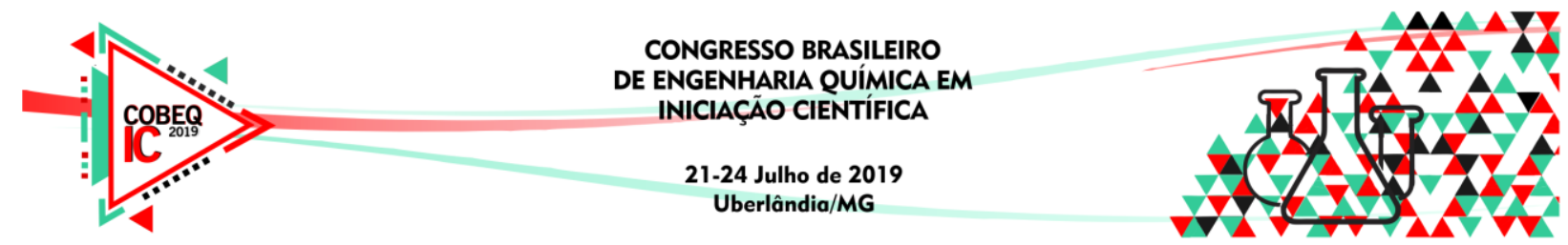

\title{
ESTUDO DA PERCOLAÇÃO ELÉTRICA DE NANOCOMPÓSITOS HÍBRIDOS DE NTC/NF/PMMA PREPARADOS VIA SOLUÇÃO
}

\author{
V. S. EVANGELISTA ${ }^{1}$, P.H.S L. COELHO ${ }^{1}$, L. SOUSA ${ }^{1}$ e J. S.PEREIRA ${ }^{1}$ \\ ${ }^{1}$ Universidade Federal do Maranhão, Departamento de Engenharia Química \\ E-mail para contato: vanessa.netbon@hotmail.com
}

\begin{abstract}
RESUMO - Neste trabalho foi realizado o estudo comparativo da condutividade elétrica de diferentes modelos de percolação para sistemas de nanocargas híbridas com negro fumo e nanotubo de carbono, dispersas em matriz de polimetilmetacrilato. Os nanocompósitos foram preparados em solução, via dispersão ultrassônica. As medidas de condutividade elétrica foram realizadas por meio da técnica de placas paralelas. Os modelos estudados são advindos da teoria do volume excluído, que avalia a sinergia em compósitos híbridos interligada com a percolação elétrica das cargas individuais. A diferença entre eles está relacionada a presença (modelo de Chen), ou não (modelo de Sun), do fator geométrico, no que tange o comportamento das cargas dispersas na formação da rede percolativa. Os estudos mostraram que a utilização da equação de Chen trouxe consideráveis vantagens para o sistema híbrido NF/NTC em relação a equação de Sun. Dentre elas, a obtenção de uma porcentagem total de cargas inferior, com um limiar de percolação que proporcionou ao sistema híbrido a mesma ordem de grandeza da condutividade elétrica, se comparado aos valores obtidos pela equação de Sun. Além disso, o modelo de Chen revelou que o negro de fumo contribuiu de forma mais eficaz para o aumento da condutividade dos nanocompósitos.
\end{abstract}

\section{INTRODUÇÃO}

Nanocompósitos poliméricos condutores representam uma nova classe de materiais obtida pela incorporação de reforço nanométrico, tal como nanotubos de carbono (NTC) e negro de fumo (NF) em matriz polimérica. As cargas de dimensões nanométricas (1-100 nm) possuem uma área de superfície elevada, promovendo melhor dispersão na matriz e por isso uma melhoria das propriedades elétricas do compósito (Bauhofer e Kovacs, 2009) Logo, estes materiais têm potencial para uso em uma variedade de aplicações como, emissão de campo elétrico, plásticos condutores, condutores térmicos, adesivos condutivos, suporte de catalisadores, aplicações biológicas, filtração de ar e água, entre outras (Ma et al., 2010). A principal questão sobre nanocompósitos poliméricos condutores, está relacionada com a variação da condutividade em função da concentração das partículas condutoras, onde se observa existir uma concentração crítica, chamada de limiar de percolação $\left(\varphi_{c}\right)$, a partir da qual ocorre um aumento na condutividade do sistema. Este fenômeno pode ser explicado pela teoria da percolação. 


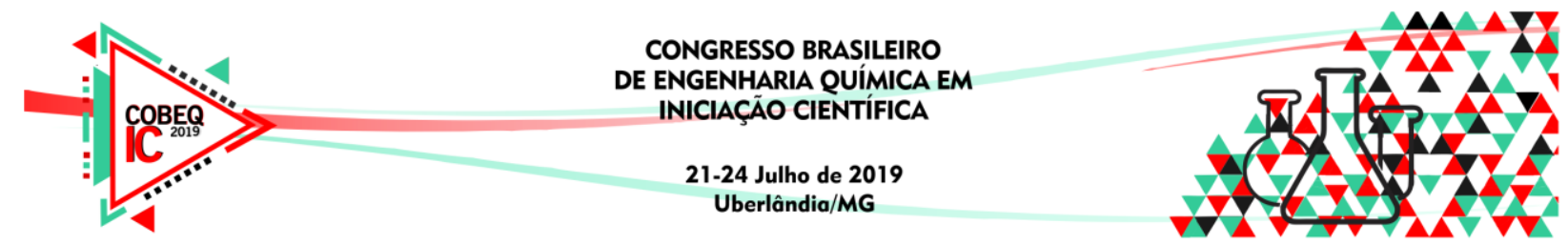

Nos últimos anos, estudos passaram a ser desenvolvidos com o intuito de analisar o comportamento elétrico dos sistemas com mais de um tipo de carga condutora, os chamados híbridos (Chen et al., 2014; Coelho et al., 2017). Para isso, alguns modelos prévios acerca desses materiais são empregados, tal como o modelo do volume excluído (Balberg et al., 1984). Para os autores o valor de $\varphi_{c}$ é inversamente proporcional ao volume excluído do objeto estudado, $\mathrm{V}_{\mathrm{ex}}$, conforme a relação descrita pela Equação 1:

$$
\varphi_{c} \propto \frac{1}{V_{e x}}
$$

Este modelo associa a relação matemática proposta para a percolação elétrica de cargas de carbono em matriz polimérica e tem sido usado como base para novos modelos que buscam o prognóstico do limiar de percolação para sistemas híbridos.

O modelo de Sun et al. (2009), desenvolvido a partir do modelo volume excluído, é útil para prever o limiar de percolação de cargas híbridas de NTC e NF, baseado na percolação das cargas individuais e nas propriedades elétricas de compósitos dispersos em matriz polimérica. A Equação 2 fornece tal modelo para sistemas contendo dois tipos diferentes de cargas condutoras A e B:

$$
\frac{V_{A}}{\varphi_{C_{2}, A}}+\frac{V_{B}}{\varphi_{C_{B} B}}=1
$$

Ademais, é notável que os aspectos geométricos das cargas individuais também influenciam de modo significativo a formação estrutural na mistura do sistema híbrido, e consequentemente no limiar da percolação elétrica (Chen et al., 2014). O modelo de Chen et al. (2013) representado pela Equação 3, indica esta sensibilidade, no que tange um limiar dependente das quantidades e das dimensões dos NTC e NF.

$$
\left(1-\frac{V_{N T C}}{\varphi_{N T C}}\right)^{1,449}+\left(1-\frac{V_{N F}}{\varphi_{N F}}\right)^{0,622+0,0531\left(\frac{D_{N F}}{D_{N T C}}\right)}=1
$$

Considerando-se o potencial para o sistema híbrido de cargas e as investigações ainda necessárias relativas aos efeitos de diferentes processos de preparação dos nanocompósitos, nível de dispersão das nanocargas e características estruturais nas propriedades elétricas, o presente trabalho é proposto. O objetivo foi o estudo da percolação elétrica de compósitos híbridos de NTC e NF em polimetacrilato de metila (PMMA) visando avaliar a hipótese do efeito de sinergismo nas propriedades elétricas, comparando-a com os principais modelos teóricos da literatura.

\section{MATERIAIS E MÉTODOS}

Na preparação dos nanocompósitos via solução, foi utilizado PMMA, Plexiglas V052, da Arkema, e Tolueno P.A.-A.C.S/SYNTH - como solvente. As cargas condutoras utilizadas foram o Nanotubo de Carbono de Paredes Múltiplas, TNM2 da Chengdu Organic Chemicals Co. Ltd., e o Negro de Fumo PRINTEX XE 2-B da Evonik Industries. Inicialmente, a solução de Tolueno/PMMA foi agitada magneticamente por 36 horas para a obtenção de uma solubilização total. Em sequência, foi realizada a determinação da quantidade de carga para a 


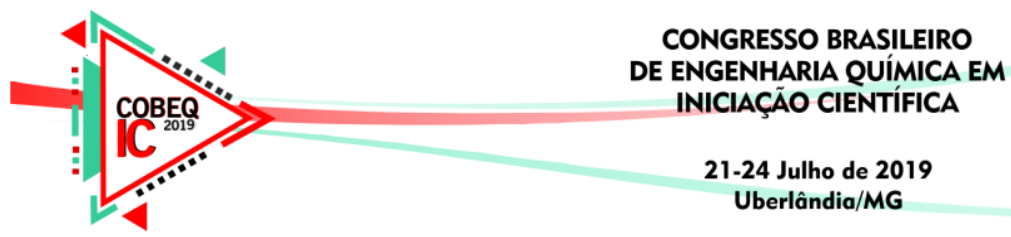

solução, Tabelas 1 e 2, respectivamente para as proporções calculadas pelas Equações 2 e 3 . Para este cálculo, foi necessário obter os valores de $\varphi_{{ }^{{ }_{N F}}}$ e $\varphi_{{ }^{{ }^{N} N C}}$ individuais.

Tabela 1 - Concentração volumétrica de Híbridos: Modelo de Sun.

\begin{tabular}{|c|c|c|c|}
\hline Amostra & NTC\% (volume) & NF \%(volume) & Total de Cargas \% \\
\hline 1-S & 0,0280 & 0,6644 & 0,6924 \\
\hline $2-\mathrm{S}$ & 0,1400 & 0,5218 & 0,6618 \\
\hline $3-\mathrm{S}$ & 0,2803 & 0,3432 & 0,6235 \\
\hline 4-S & 0,4201 & 0,1642 & 0,5852 \\
\hline
\end{tabular}

Os resultados para estes sistemas foram determinados pelo grupo de pesquisa em estudos anteriores, obtendo $\varphi_{{ } N T C}=0,55$ e $\varphi^{{ }{ }_{N F}}=0,70$ de percolação.

Tabela 2 - Concentração volumétrica de Híbridos: Modelo de Chen.

\begin{tabular}{|c|c|c|c|}
\hline Amostra & NTC\% (volume) & NF \%(volume) & Total de Cargas \% \\
\hline 1-C & 0,0280 & 0,5521 & 0,5801 \\
\hline 2-C & 0,1400 & 0,3268 & 0,4668 \\
\hline 3-C & 0,2803 & 0,1610 & 0,4413 \\
\hline 4-C & 0,4201 & 0,0522 & 0,4731 \\
\hline 5-C & 0,0025 & 0,6645 & 0,6669 \\
\hline 6-C & 0,0385 & 0,5218 & 0,5603 \\
\hline 7-C & 0,1291 & 0,3432 & 0,4723 \\
\hline 8-C & 0,2770 & 0,1642 & 0,4412 \\
\hline
\end{tabular}

Foi realizada a dispersão prévia da solução de tolueno/cargas híbridas com proporção 1:1, por 1 hora, em banho ultrassônico da marca UNIQUE USC - 185. A mistura das duas soluções, resultando em tolueno/PMMA/cargas híbridas foi submetida à ultrassom durante 30 minutos com 35\% de potência em equipamento UNIQUE - DES500. A mistura foi depositada em uma placa metálica, $75 \mathrm{~mm}$ de diâmetro, e os filmes dos compósitos foram obtidos por evaporação solvente, à temperatura ambiente. A medição de condutividade elétrica dos híbridos foi realizada através do método de placas paralelas, em equipamento KEITHLEY 2401 Source meter.

\section{RESULTADOS E DISCUSSÕES}

Pode-se observar na Figura 1 que a fração total de cargas híbridas necessárias para atingir o limiar de percolação dos híbridos, utilizando o modelo de Chen, foi inferior ao modelo proposto por Sun. Isto deve-se ao fato do modelo de Chen levar em consideração a possibilidade de conexão geométrica entre as partículas, para a obtenção de um limiar de percolação mais fiel à realidade experimental (Chen et al., 2014). A área entre as curvas 1-4S e 1-4C mostra o efeito sinérgico presente no modelo de Chen, que acontece quando $\mathrm{V}_{\mathrm{NTC}} / \varphi_{\mathrm{c}, \mathrm{NTC}}+\mathrm{V}_{\mathrm{NF}} / \varphi_{\mathrm{c}, \mathrm{NF}}<1$, no modelo de Sun, evidenciando a importância da geometria das cargas, principalmente quando uma delas possui alta razão de aspecto. Este resultado experimental do efeito sinérgico foi estudado por Coelho et al. (2017) e mostrou que concentrações inferiores ao modelo de Sun, levaram à formação de nanocompósitos 


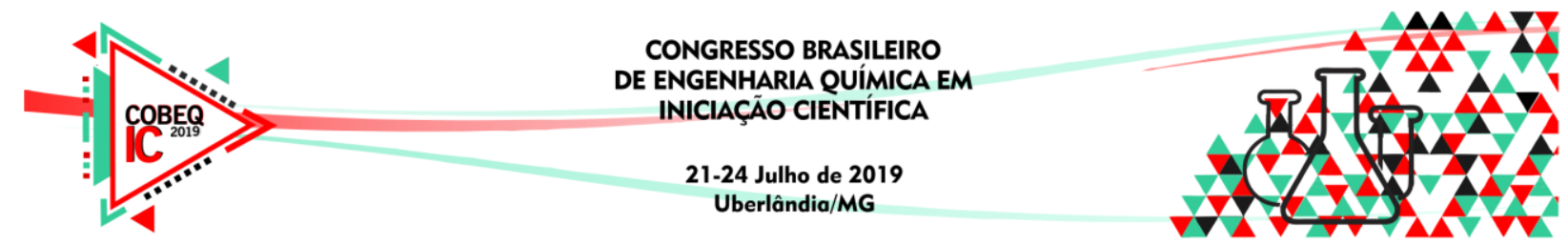

semicondutores, mediante à uma fina dispersão de cargas e à uma boa interação das diferentes geometrias, fator este fundamental para condutividade de sistemas híbridos (Li et al., 2007).

Figura 1 - Frações volumétricas de NTC e NF calculadas pelos modelos de Chen e Sun.

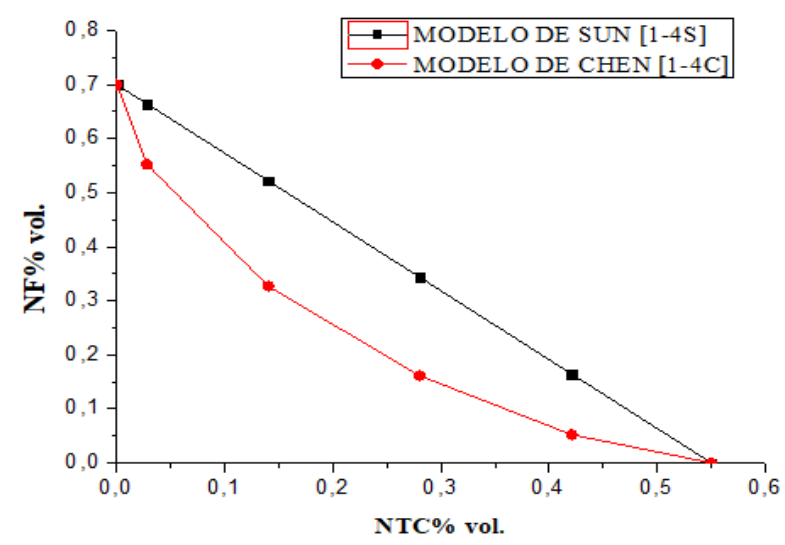

A Figura 2 mostra que os resultados gerais de condutividade obtidos para os 2 modelos deslocaram a condutividade do polímero, próxima de $10^{-11} \mathrm{~S} / \mathrm{m}$, em até 6 ordens de grandeza, formando nanocompósitos semicondutores com pequenas frações volumétricas totais de carga, abaixo de $0,7 \%$.

Figura 2 - Comparação da condutividade elétrica dos diferentes modelos de sistemas híbridos pela fração volumétrica de NTC.

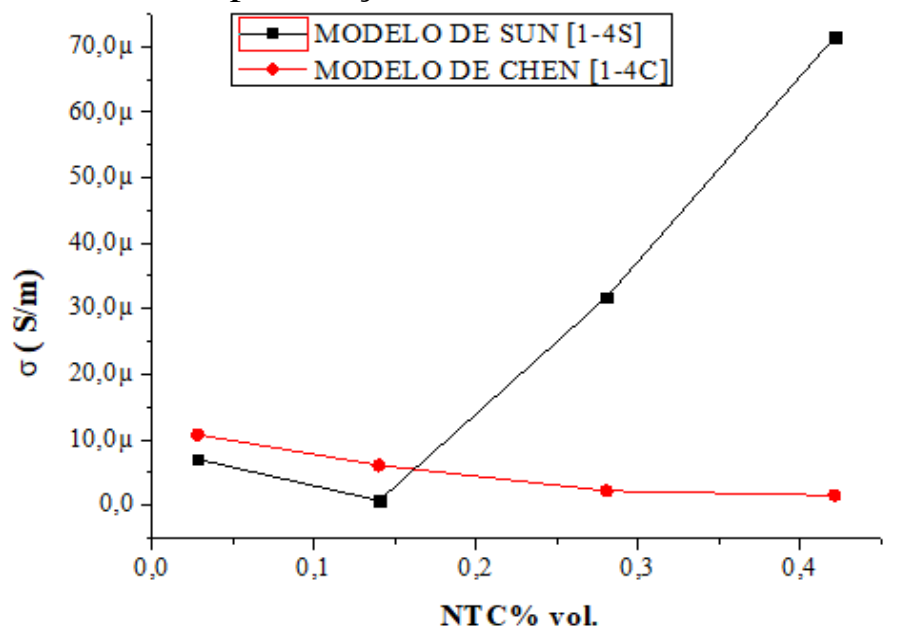

Apesar dos valores de condutividade obtidos serem próximos, é perceptível uma maior disparidade entre as amostras 3S e 3C, e as amostras 4S e 4C. Pelas tabelas 1 e 2, é possível avaliar que as concentrações de NF para o modelo de Chen são inferiores se comparadas ao modelo de Sun, influenciando na concentração total de nanocargas que reduz em até 23,5\% para as amostras de 1-4C. Sabe-se que em uma matriz híbrida de NF e NTC é necessário uma fração inferior de NTC em relação ao NF, para se obter compósitos condutores, pois devido a distância de tunelamento necessária para a passagens da corrente elétrica, apenas uma certa quantia NTC está envolvida no transporte de carga (Chen et al., 2013; Szeluga et al., 2015), o que parece não acontecer com 3C e 4C, que mantém proporções superiores de NTC em 


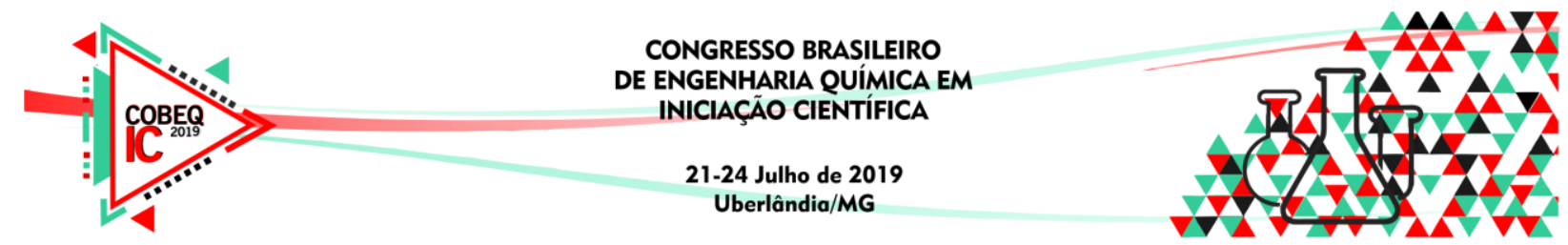

relação a NF, evidenciando um possível prejuízo sinérgico, e diminuindo a condutividade. Por este fato, analisando o modelo de Chen, podemos sugerir NF contribuiu de forma mais eficaz para o aumento da condutividade dos sistemas.

As Figuras 1 e 2 mostram que a análise da estrutura das nanocargas de NF e NTC na matriz polimérica proposta pelo modelo de Chen, de fato traz uma sensibilidade para o comportamento elétrico do compósito. Deve-se ter em vista, que a disposição estrutural dos NTC na matriz é influenciada pelas nanopartículas de NF, se a dispersão é suficiente, e se estas possuírem aglomerações similares. É fato que as partículas de NF atuam como "espaçadores", e a sinergia decorre da ligação eficaz das lacunas do NF preenchidas pelas longas e flexíveis conformações do NTC, atuando como pontes condutoras de elétrons (Szeluga et al., 2015). De forma similar, o aumento na concentração de NF nos nanocompósitos híbridos leva à formação de novos caminhos condutores entre cargas NTC. De acordo com Li et al. (2007), a distância de tunelamento entre NTC determina a condutividade global em compósitos com matriz polimérica. Sumfleth et al. (2008) estudou o comportamento elétrico de compósitos híbridos de NF/NTC, e deduziu que o aumento da condutividade resulta diretamente da redução de tunelamento das distâncias entre as partículas de NF dispostas entre os entrelaçados de NTC. A Figura 3 mostra exatamente esta relação, pois as amostras de 5-8C, calculadas pela variação de concentrações de $\mathrm{NF}$ e obtenção de concentrações de NTC isolando o termo correspondente da Equação 2, apresentaram maiores condutividades para maiores concentrações de NF, em relação as amostras de 1-4C, comportamento este, também observado na Figura 2.

Figura 3 - Condutividade elétrica dos híbridos pela fração volumétrica de NTC utilizando o modelo de Chen.

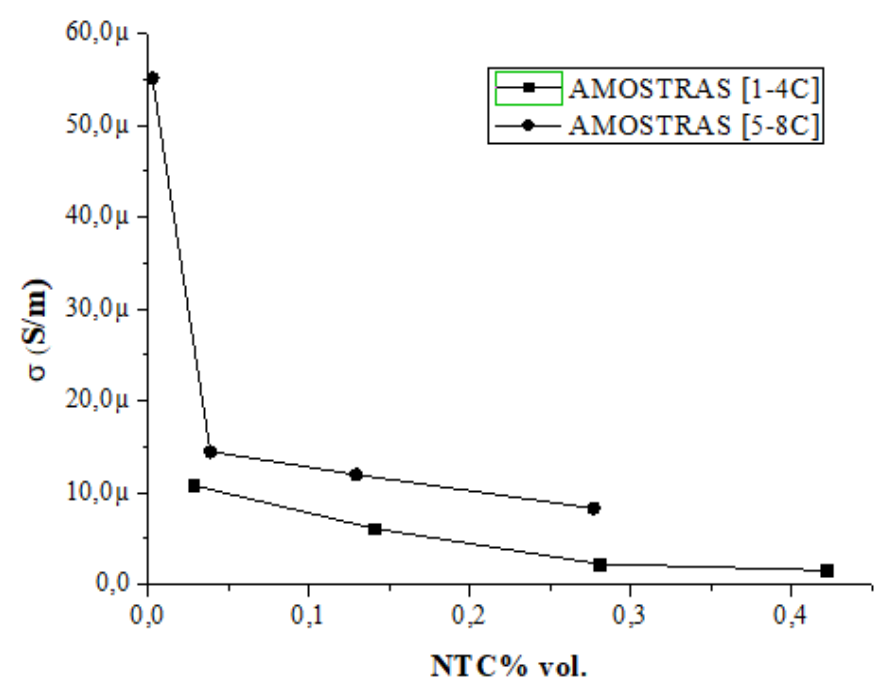

\section{CONCLUSÃ̃o}

Na comparação dos modelos, a equação de Chen mostrou que a inclusão da dimensão geométrica das nanocargas foi favorável à redução da quantidade total necessária para a percolação elétrica de cada amostra, inferindo um menor custo-benefício para futuras aplicações destes sistemas híbridos. Além disso, esta redução, não influenciou de forma significativa na condutividade elétrica dos nanocompósitos, haja visto, que os semicondutores 


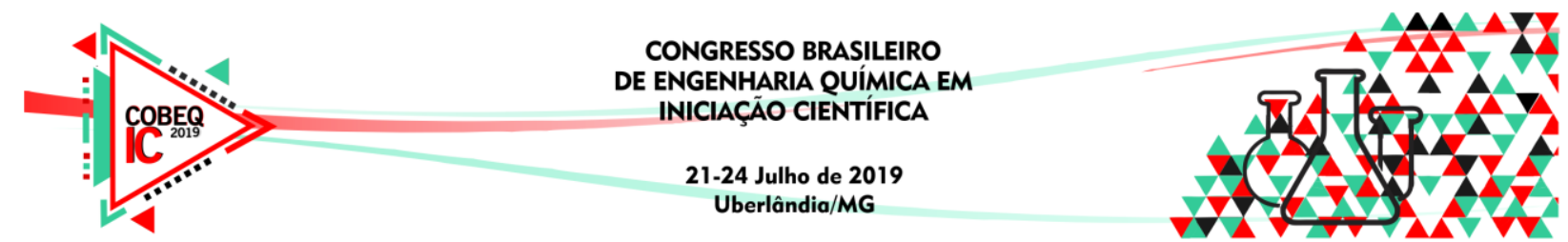

formados obtiveram propriedades semelhantes aos experimentos calculados com o modelo de Sun. A avaliação específica do modelo de Chen, revelou que, entre as cargas condutoras que formam o sistema híbrido, o NF contribuiu de forma mais eficaz para o aumento da condutividade dos nanocompósitos.

\section{NOMENCLATURA}

V - Fração volumétrica de carga; $\varphi$ - Limiar de percolação elétrica; D - Diâmetro da carga.

\section{REFERÊNCIAS}

BALBERG, I.; ANDERSON, C. H.; ALEXANDER, S. \& WAGNER, N. Excluded volume and its relation to the onset of percolation. Physical Review B, 30(7), p. 3933-3943, 1984.

BAUHOFER, W. \& KOVACS, J. Z. A review and analysis of electrical percolation in carbon nanotube polymer composites. Composites Science and Technology, 69(10), p. 14861498, 2009.

CHEN, J.; DU, X-C.; ZHANG, W-B.; YANG, J-H.; ZHANG, N.; HUANG, T. \& WANG, Y. Synergistic effect of carbon nanotubes and carbon black on electrical conductivity of PA6/ABS blend. Composites Science and Technology, 81, p. 1-8, 2013.

CHEN, Y.; WANG, S.; PAN, F. \& ZANGHET, J. A Numerical Study on Electrical Percolation of Polymer-Matrix Composites with Hybrid Fillers of Carbon Nanotubes and Carbon Black. Journal of Nanomaterials. P. 614797-614806, 2014.

COELHO P-L.; ARMELLINI, V. A. D. \& MORALES, A. R. Assessment of percolation threshold simulation for individual and hybrid nanocomposites of carbon nanotubes and carbon black. Materials Research, 20 (6), p.1638-1649, 2017.

LI, C; THOSTENSON, E. T. \& CHOU, T. W. Dominant role of tunneling resistance in the electrical conductivity of carbon nanotube-based composites. Applied Physics Letters, 91, p. 223114, 2007.

MA. P-C.; SIDDIQUI, N. A.; MAROM, G. J. \& KIM, K. Dispersion and functionalization of carbon nanotubes for polymer-based nanocomposites: A review. Composites Part A: Applied Science and Manufacturing (41), p.1345, 2010.

SUMFLETH, J.; ALMEIDA, P-S.; SRIYAI, M. \& SCHULTE, K. Titania-doped multiwalled carbon nanotubes epoxy composites: enhanced dispersion and synergistic effects in multiphase nanocomposites. Polymer, 49, p. 5105-5112, 2008.

SUN, Y.; BAO, H.; GUO, Z.X. \& Yu, J. Modeling of the electrical percolation of mixed carbon fillers in polymer-based composites. Macromolecules, 42, p. 459-63, 2009.

SZELUGA, U., KUMANEK B., TRZEBICKA, B. Synergy in hybrid polymer/nanocarbon composites. A review. Composites: Part A, 73, p. 204-231, 2015. 